\title{
Epidermal Growth Factor (EGF) induces a phenotypic switch of a human glioblastoma cell line to neurospheres.
}

\author{
T. Bargar*, H. El-Refaey,*, I. Ahmad, **, M. Ebadi*** and J. Rodriguez-Sierra* \\ * Dept. Cell Biology and Anatomy, Univ. Nebraska Med. Ctr. (UNMC), Omaha, NE 68198-6395 \\ ** Dept. Ophthalmology, UNMC, Omaha, NE 68198-7691 \\ *** Dept. Pharmacology and Toxicology, Univ. North Dakota, Grand Forks, ND 58202-9037
}

Recently there has been an enormous amount of interest on the transformation of cells from thei $r$ original phenotype to a more or less differentiated cell type. The work with stem cells has gained national and international prominence due to the potential for therapeutic treatments that this work promises. The work on cells of the nervous system ha $\quad \mathrm{s}$ been one of the major sources for controversy and a very recent issue of the Journal of Neuroscience [1][2] attempted to clarify some of the issues by publishing a series of mini-reviews in this area. The human glial cell line U-373 MG (Astrocytoma. Grade III, human) was phenotyped using immuno -histochemical methods. We typed the cells as astrocytes using antisera to GFAP (glial fibrillary acidic protein, see Fig 1). Our original cell culture medium was RPMI -1640 (Gibco Labs). We found that by altering the media by adding $\mathrm{EGF}_{2 \alpha}(20 \mathrm{ng} / \mathrm{ml})$ and re-feeding the cells every 4 days , we saw the cells gradually altering their morphology and their phenotype when tested by immunochemical methods.

This current study will focus in describing the major morphological changes in the cells using Scanning Electron Microscopy (SEM). We used the JEOL T -220A SEM to analyze our specimens. All cells were plated onto plastic cover slips (NUNC), the cell s were rinsed of media and fixed with $2.5 \%$ glutaraldehyde in 0.1 M Sorensen Phosphate buffer ( $\mathrm{pH}$ 7.2). The fixed cells were dehydrated in an ethanol series and were critically point-dried in a Pelco CPD2 and coated with gold palladium (45nm thick) using a Hummer VI Sputter Coater.

We decided to try to see if a human glioblastoma cell line could b e made to alter its phenotype by treating these cells with a Growth factor that is a potent proliferative as well as a differentiating agent, EGF. Before the cells were treated with EGF, the cells plated very well and efficiently to the culture dishes and displayed morphological characteristic of glial cells grown in culture (see Fig. 2). Four days after EGF treatment, we began to see some differences in the shape and features of the cells and the cells appear more heterogenous, some cells changing their shape drastically to become rounder and by produ cing small spheres that grew on top of the cells that were attached to the substrate (see Fig. 3). After the second feeding with EGF, the cells began to change the architecture of their growth pattern, resem bling a checker board with elongated cells forming a lattice and the small spheres growing on the corners of the lattice (see Fig. 4 and 5 ). After the $4^{\text {th }}$ and $5^{\text {th }}$ feeding time, the spheres began to form multi -layered 3 -dimensional structures and sometimes float away from the other cells (see Fig. 6). These cells after continued feeding began to express Nestin (a stem cell marker for neurons) and eventually expressed the NMDA receptor and MAP1 protein.

We hope that by describing the morphological altera tions that these cells undergo, we can explain the sequence of events that lead to the phenotypic alteration of a cell and might provide others with some hints about what to expect in cells undergoing similar changes. 
References

[1] F. Gage., J. Neuroscience.22 (2002) 612.

[2] C. Kintner., J. Neuroscience.22 (2002) 639

[3] This research was supported by the NIH, NIA AG-17059 and funds from the Nebraska Research initiative and a grant from the UNMC Dean's Indirect Costs.

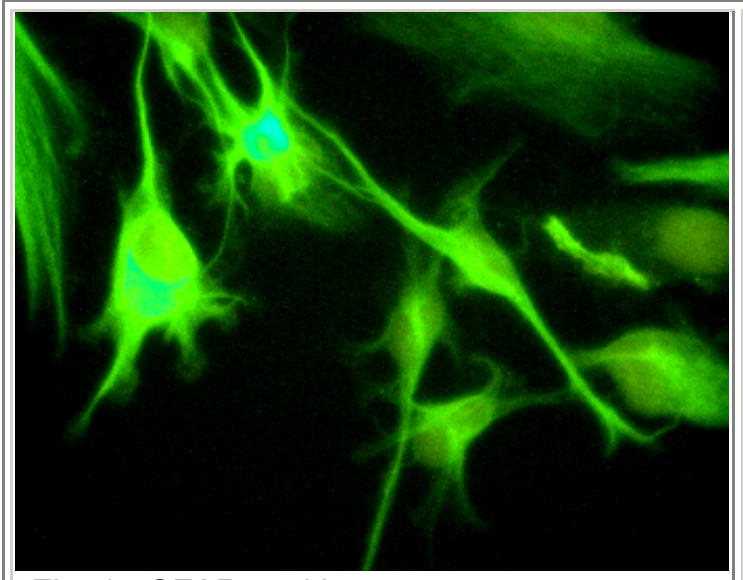

Fig. 1. GFAP positive cells

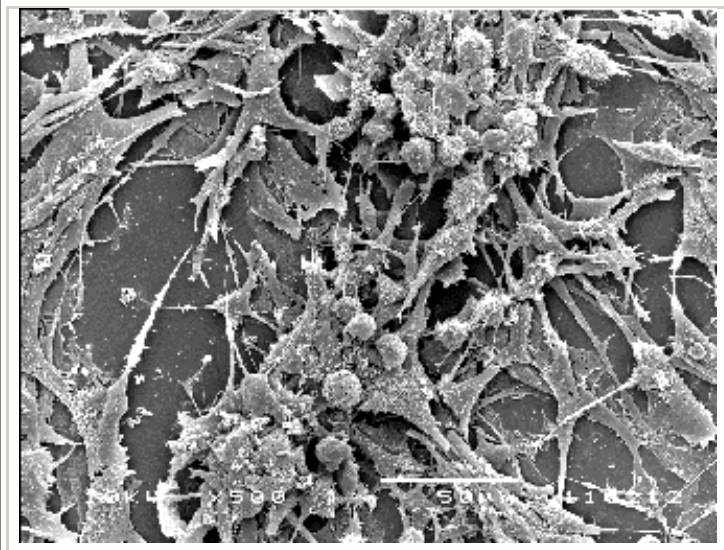

Fig. 3. After 4 days of EGF

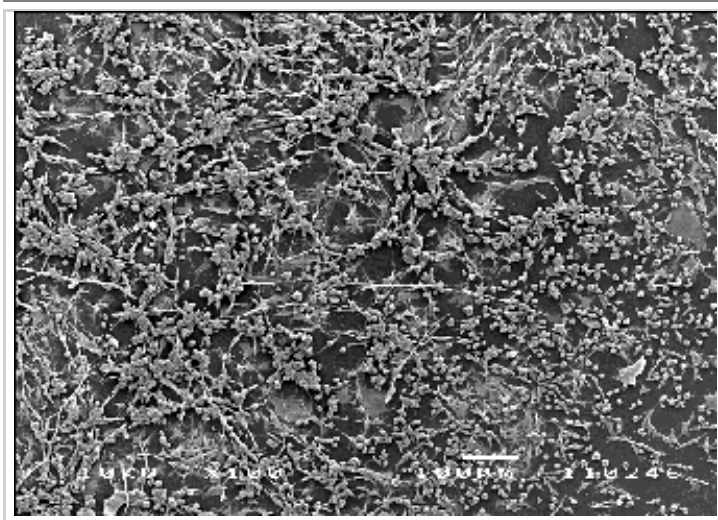

Fig. 5 After 12 days of EGF

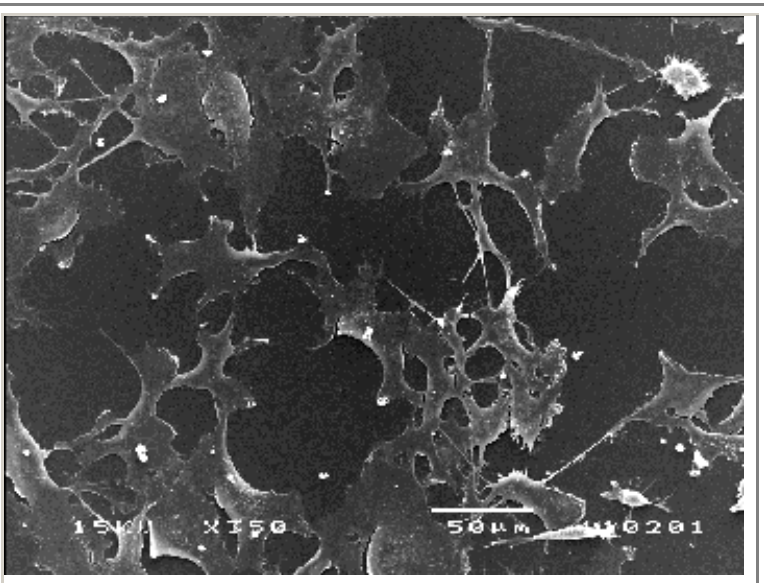

Fig. 2. Glial cells prior to EGF

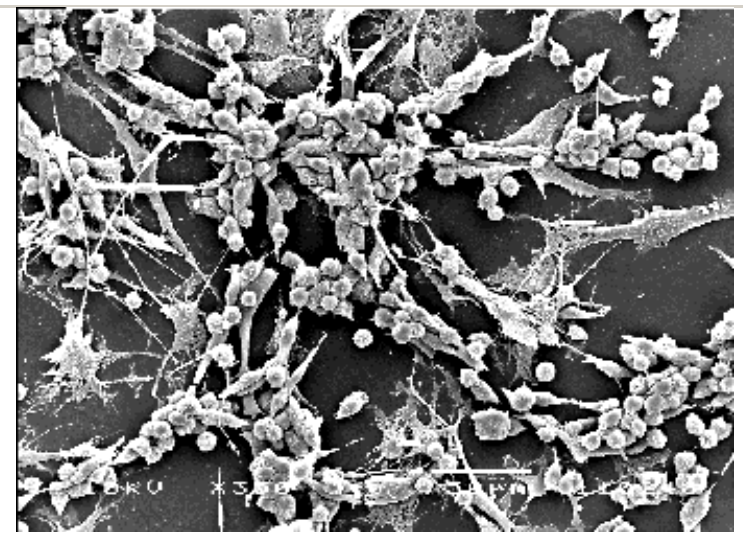

Fig. 4 After 8 days of EGF

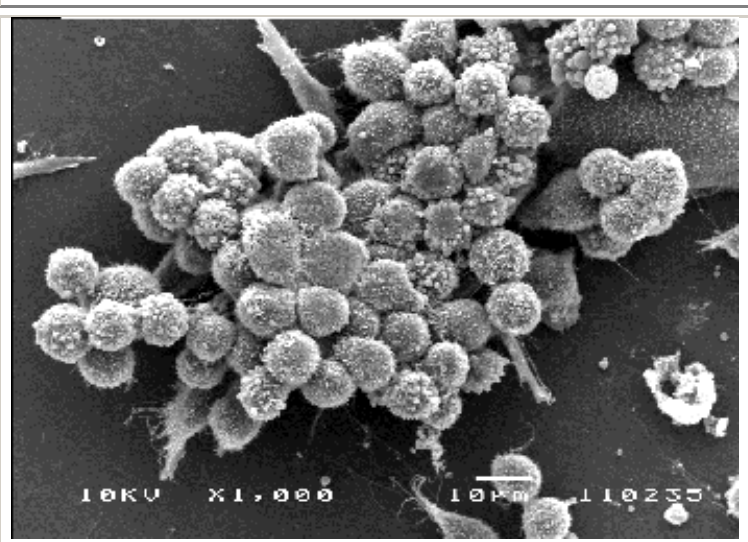

Fig. 6. Spheres formed after 8 days of EGF 